\title{
Modeling the Effects of $O$-sulfonation on the CID of Serine
}

\author{
Kenneth Lucas and George L. Barnes* \\ Department of Chemistry and Biochemistry \\ Siena College \\ 515 Loudon Road \\ Loudonville, NY 12211 \\ E-mail: gbarnes@siena.edu
}

\begin{abstract}
We present the results of direct dynamics simulations and DFT calculations aimed at elucidating the effect of $O$-sulfonation on the collision induced dissociation for serine. Towards this end, direct dynamics simulations of both serine and sulfoserine were performed at multiple collision energies and theoretical mass spectra obtained. Comparisons to experimental results are favorable for both systems. Peaks related to the sulfo group are identified and the reaction dynamics explored. In particular, three significant peaks $(\mathrm{m} / \mathrm{z}, 106,88$, and $81)$ seen in the theoretical mass spectrum directly related to the sulfo group are analyzed as well as major peaks shared by both systems. Our analysis shows that the $m / z, 106$ peaks result from intramolecular rearrangements, intermolecular proton transfer among complexes composed of initial fragmentation products, and at high energy sidechain fragmentation. The $m / z 88$ peak was found to contain multiple constitutional isomers, including a previously unconsidered, low energy structure. It was also seen that the RM1 semi empirical method was not able to obtain all of the major peaks seen in experiment for sulfoserine. In contrast, PM6 did obtain all major experimental peaks.
\end{abstract}

\section{Introduction}

Significant experimental ${ }^{1-5}$ and theoretical ${ }^{6,7}$ work has been performed to examine both the chemical and physical aspects of MS2 systems. It is known that the addition of post-translational modifications (PTM) can expand the functional diversity of proteins and peptides by altering their configurational space, ${ }^{8}$ enzymatic efficiency, ${ }^{9}$ signaling properties, ${ }^{10}$ and a myriad of other biochemically important criteria. ${ }^{11}$ Such PTMs can occur at the N-terminus, C-terminus, side chain, or backbone depending on the modification and the residue. The phosphate modification, particularly $O$-phosphorylation of threonine and serine, is ubiquitous and has long been studied. ${ }^{12,13}$ However, the sulfate analog was only recently first described in the literature in $2004,{ }^{14}$ and as such, much less work has been done on peptide sulfonation, especially within the realm of proteomics, though some initial work has been performed. ${ }^{15,16}$

Recently Polfer and co-workers have studied the MS2 spectra of $O$-sulfonated serine (sulfoserine $\mathrm{m} / \mathrm{z}$ 186) through both collision induced dissociation (CID) mass spectrometry and infrared multiple photon dissociation (IRMPD) spectroscopy. ${ }^{17}$ The CID measurements found that there were two major fragmentation pathways, one associated with $\mathrm{SO}_{3}$ loss $(\mathrm{m} / z$ 106) and one associated with $\mathrm{H}_{2} \mathrm{SO}_{4}$ loss $(\mathrm{m} / \mathrm{z}$ 88). In addition, DFT calculations were used along with the IRMPD spectra to infer product structures. Proposed mechanisms 
were also provided based on chemical intuition.

Molecular dynamics (MD) simulations have been previously used to investigate the fragmentation pathways of unmodified amino acids and peptides and are known to yield results in reasonable agreement with experiment. ${ }^{6,7}$ Performing MD simulations of gas phase amino acids and peptides has lent valuable insight into the complicated, short-timescale fragmentation dynamics taking place during CID, which often follow unexpected reaction pathways. The calculation of an ensemble of trajectories that simulate random collisions of the ion species of interest and the subsequent dynamics allows for a guided approach to the investigation of the fragmentation mechanisms via $a b$ initio calculations. Hence, it is expected that a direct dynamics/DFT study would provide insight into the sulfoserine system.

In this work, we will investigate both the serine and sulfoserine system using a quantum mechanical (QM) + molecular mechanical (MM) direct dynamics approach followed by high level DFT calculations to accurately quantify the relevant stationary points along the most important reaction pathways. This will allow for an evaluation of the effect of PTM on the mechanism as well as a comparison of our short-time results to the long-time results of Polfer.

An outline for the remainder of the paper is as follows: in Section 2, we provide an overview of our computational method; in Section 3, we present our results and discuss what insight our calculations provide regarding the reaction dynamics; and in Section 4, we provide a summary.

\section{Computational Methods}

The use of direct dynamics simulations to investigate MS2 systems has been well described in the literature, ${ }^{6,18,19}$ and hence, only a summary is provided here. The interested reader is directed to the recent tutorial review. ${ }^{6}$ Below, we will describe our treatment of the potential energy function used for the dynamics simulations, how the starting structure for sulfoserine was obtained, and an overview of the initial conditions. Our analysis method is then described.

\subsection{Potential Energy}

Following the established method, we write our potential energy function for the collision system as a sum of intramolecular and intermolecular terms. Specifically, the potential energy is given by

$$
V=V_{\text {peptide }}+V_{\text {Ar-peptide }}
$$

where $V_{\text {peptide }}$ is the intramolecular potential of the peptide (serine or sulfoserine) and $V_{A r-p e p t i d e}$ is the intermolecular potential. In this work, we choose to use semi-empirical methods to calculate $V_{\text {peptide }}$. In particular, we investigated both RM1 and PM6 initially. RM1 has been shown to provide good results in simulations; ${ }^{19-25}$ however, we found that it lacked some important mass peaks that PM6 was able to obtain. A similar deficiency was seen in the recent simulations of Macaluso et $a l^{26}$ in which it was found that PM6D outperformed RM1. To our knowledge, these are the only two direct dynamics simulations that have employed RM1 for systems containing sulfo groups, and both works concluded that a version of PM6 was superior. Given that it is now known that RM1 is deficient for sulfo containing species, we will focus our discussion on the PM6 results.

The intermolecular potential, $V_{\text {Ar-peptide }}$, is calculated as the sum of two-body Buckingham potential terms between argon and the atoms in the amino acid, expressed as

$$
V_{\text {Ar-peptide }}=A_{A r-k} e^{-B_{A r-k} R_{A r-k}}+\frac{C_{A r-k}}{R_{A r-k}^{9}}
$$

where the index $k$ represents an atom within the amino acid, and $R_{A r-k}$ is the interatomic distance. The parameters for the intermolecular interactions with $\mathrm{C}, \mathrm{N}, \mathrm{O}$ and $\mathrm{H}$ were developed by Meroueh and Hase, ${ }^{27}$ while those for the terms involving the $\mathrm{SO}_{4}$ moiety were parameterized by Oritz and Salpin. ${ }^{28}$ These parameters result in an intermolecular potential that is strictly repulsive, which is sufficient for our simulations.

\subsection{Structures and Simulation Method}

We generated initial structures using Avogadro ${ }^{29}$ followed by optimization from Mopac2016. ${ }^{30}$ Once optimized, the peptide was given a random 
orientation about its center of mass along with a $300 \mathrm{~K}$ vibrational and rotational distribution using normal mode sampling. ${ }^{31}$ The impact parameter was randomly chosen between 0 and $3 \AA$, and the argon atom was situated $20 \AA$ from the center of mass of the molecule, which ensures no initial interaction. A total of 1,500 trajectories were calculated for each collision energy between 2.00 and $11.00 \mathrm{eV}$ at $1.50 \mathrm{eV}$ increments. We solved Hamiltonian equations of motion for each trajectory using a $6^{\text {th }}$ order symplectic integration scheme ${ }^{32}$ for total simulation time of 50 ps with a 1 fs step size and output written every $50 \mathrm{fs}$. This was accomplished via an in-house simulation package coupled with Mopac2012. ${ }^{33}$ Following the collision, argon was removed when the MM interaction $V_{\text {Ar-peptide }}$ fell below $1 \times 10^{-3}$ $\mathrm{kcal} / \mathrm{mol}$. In addition, since only charged fragments are observed in experiment, neutral fragments were removed from the simulation if they were at least $15 \AA$ away from any charged fragments. This removal also improved computational efficiency and self-consistent field convergence. Preliminary simulations also revealed that for the serine/sulfoserine system, secondary fragmentation did not occur for charged species with $\mathrm{m} / \mathrm{z} \leq 60$. Hence, simulations were terminated if the charged fragment was below this cutoff. Moreover, experimental observation of low mass products can be problematic and ultimately our goal is to provide information relevant to experiment. Energy was well conserved for all trajectories.

\subsection{Theoretical Mass Spectra and Re- action Mechanisms}

Our in-house simulation package calculates bond orders between all QM atoms for all steps within the simulation. With this information, both the connectivity and the number of fragments is available for each simulation step. This in turn allows for theoretical mass spectra to be automatically constructed for both species as well as providing data for a group theory analysis of the products that contribute to each peak. ${ }^{34,35}$ With this knowledge of the final products, as well as the intermediates, we generated structures that are close to the minima, intermediates, and transition states of interest, and proceeded to optimize them at the $\omega \mathrm{B} 97 \mathrm{X}-\mathrm{D} /$ aug-cc-pVTZ level of theory using Gaussian09 software. ${ }^{36}$

\section{Results and Discussion}

\subsection{Serine Mass Spectrum}

We begin our discussion by examining the results for the comparatively simple serine system. The theoretical mass spectrum for serine at a collision energy of $8 \mathrm{eV}$ is displayed in Figure 1, and it is seen that the spectrum is dominated by the $m / z 60$ peak at all collision energies considered. This peak corresponds to the loss of $\mathrm{H}_{2} \mathrm{O}+\mathrm{CO}$ or $\mathrm{C}(\mathrm{OH})_{2}$. Roughly 55\% yield the first product at $8 \mathrm{eV}$. This product is produced via two pathways: 1) Loss of water occurring at the $\mathrm{C}$-terminus as a result of a proton hop from the $\mathrm{N}$-terminus and shortly thereafter loss of $\mathrm{CO}$ from the $\mathrm{C}$-terminus, and 2) Loss of $\mathrm{C}(\mathrm{OH})_{2}$ occurs via a proton hop to the carbonyl oxygen from the $\mathrm{N}$-terminus that results in the $\mathrm{C}$ terminus dissociating. Initial attempts to optimize the transition states associated with the loss of the C-terminus were unsuccessful largely due to the locally shallow PES. We also observe the $m / z, 88$ peak, which is associated with the loss of water, and occurs through two different processes. Water is lost during the first step for one of the pathways for the formation of $m / z 60$, and hence $m / z 88$ is an intermediate. It likely that with additional simulation time, such intermediates would react to $\mathrm{m} / \mathrm{z} 60$ as well. The second process for the formation of $\mathrm{m} / \mathrm{z} 88$ is for a proton to migrate from the $\mathrm{N}$-terminus to the alcohol of the side chain followed by water dissociation. There is one additional minor peak, $\mathrm{m} / \mathrm{z} 76$, which corresponds to loss of the side chain without proton migration occurring. Our theoretical results are in good qualitative agreement with the work of Zhang et $a l^{37}$ though we do not see the $\mathrm{m} / \mathrm{z} 70$ peak, which is attributed to the loss of two waters.

\subsection{Sulfoserine Mass Spectrum}

Turning our attention to sulfoserine, the theoretical mass spectrum at a collision energy of $8 \mathrm{eV}$ along with the collision energy dependence of the major product peaks is shown in Figure 2. The 

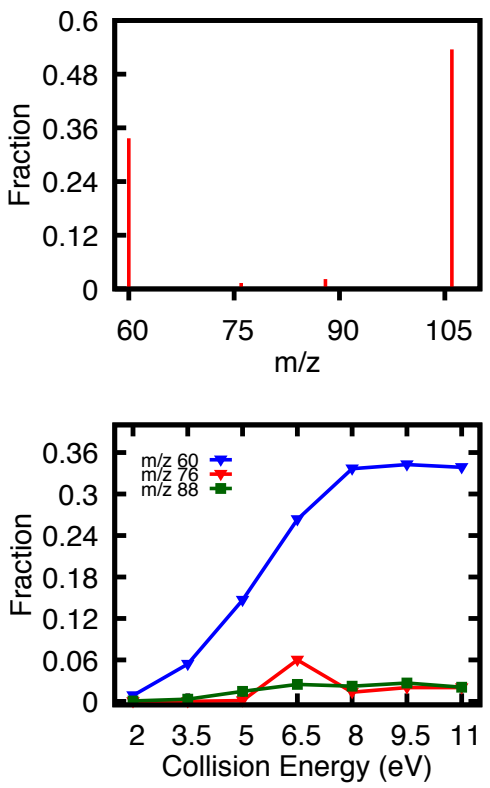

Figure 1: An example theoretical mass spectrum obtained from a collision energy of $8 \mathrm{eV}$ for serine along with the collision energy dependence of the major product peaks. It is seen that at this collision energy and within the time frame of the simulation, the majority of trajectories do not react. The most significant reactant peak occurs at $\mathrm{m} / \mathrm{z} 60$, which corresponds to $\mathrm{H}_{2} \mathrm{O}+\mathrm{CO}$ or $\mathrm{C}(\mathrm{OH})_{2}$ loss.

four most significant peaks are $m / z, 140,106,88$, and 81 which correspond to loss of $\mathrm{H}_{2} \mathrm{O}+\mathrm{CO}$ or $\mathrm{C}(\mathrm{OH})_{2}$, loss of $\mathrm{SO}_{3}$, loss of $\mathrm{H}_{2} \mathrm{SO}_{4}$, and formation of $\mathrm{HSO}_{3}^{+}$, respectively. These results are in good qualitative agreement with Polfer and coworkers ${ }^{17}$ with the exception that $m / z, 129$ was not observed in our spectrum and $m / z, 81$ was not observed in the experiment. The $m / z, 129$ is a minor peak that was not analyzed, while $m / z 81$ grows in intensity with collision energy and will be discussed below. Of the four peaks observed (140, 106, 88, and 81) in our simulations, the last three peaks all involve the PTM, while the first follows an equivalent mechanism as that seen in unmodified serine: loss of either $\mathrm{H}_{2} \mathrm{O}+\mathrm{CO}$ or $\mathrm{C}(\mathrm{OH})_{2}$. The former occurs $65 \%$ of the time, making it slightly more probable than in unmodified serine.

\subsubsection{Sulfoserine $m / z, 106$}

Connectivity analysis of the trajectories yielding the $m / z, 106$ fragment ion was performed and
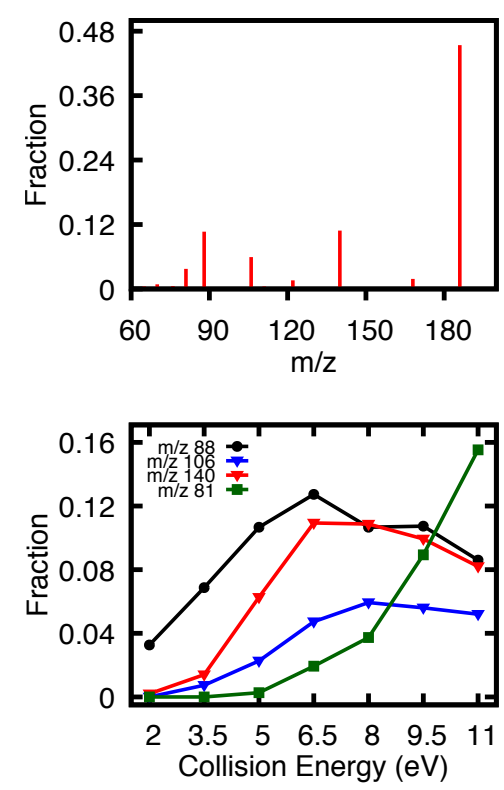

Figure 2: An example theoretical mass spectrum obtained from a collision energy of $8 \mathrm{eV}$ along with the collision energy dependence of the major product peaks. It is seen that at this collision energy and within the time frame of the simulation, the majority of trajectories do not react. The most significant reactant peaks are $\mathrm{m} / \mathrm{z}$ 140, 106, 88 and $81 . m / z 81$ is seen to dramatically increase in intensity at large collision energies. The $m / z 88$ and 106 peaks are the dominant peaks seen in experimental work of Polfer and co-workers.

revealed that protonated serine is the dominant species in the peak. This agrees with previous experimental work by Polfer and coworkers. ${ }^{17}$ However, that work only proposed one mechanism for its formation, whereas multiple mechanisms were observed in the simulations. Moreover, there are three different classes of mechanisms that can form the $\mathrm{m} / \mathrm{z} 106$ peak: 1) Intramolecular rearrangement reactions, such as those shown in Scheme 1, 2) Intermolecular proton transfer reactions that take place due to complexation of fragmentation products, and 3) "Shattering" mechanisms that are rare and only occur at large collision energies. As an aside, we note that the theoretical spectrum resulting from simulations using RM1 did not contain the $m / z 106$ peak, and hence, the method was deemed insufficient for this system. Since $m / z 106$ results from cleavage of the $\mathrm{S}-\mathrm{O}$ bond, it can be assumed that RM1 overesti- 
mates such bond strengths.

The first class of mechanisms are shown schematically in Scheme 1. Polfer and coworkers proposed Mechanism 106-A, which produces $\mathrm{SO}_{3}$ from sulfoserine in a concerted step by transferring the sulfate proton to the linker oxygen of the side chain. In our simulations, this reaction pathway dominates the $m / z, 106$ peak for all collision energies. The lowest fraction of occurrence for the pathway is 0.66 at a collision energy of 8 $\mathrm{eV}$, whereas the largest fraction is one. DFT calculations estimate that the barrier for this reaction is $38.1 \mathrm{kcal} / \mathrm{mol}$ and involves the migration of a single proton. While this pathway is dominant, there are other intramolecular rearrangements that can occur. In particular, it is possible for the excess proton on the $\mathrm{N}$-terminus to become involved as shown in Mechanism 106-B: a proton transfer occurs from the N-terminus to the side chain oxygen followed by a transfer from the sulfate group back to the $\mathrm{N}$-terminus, releasing $\mathrm{SO}_{3}$ and generating $\mathrm{N}$-protonated serine. This mechanism can take place in two different ways, depending on the conformation present. If the conformation has a short $\mathrm{N}$ to $\mathrm{O}$ distance, then there is no energy minimum corresponding to the first step. This single well character is similar to that seen in low barrier hydrogen bonds (also called short strong hydrogen bonds), ${ }^{38}$ a class of hydrogen bond that have been found to be important for proton transfer in biological systems. ${ }^{39}$ As such, the first proton transfer can take place along the intrinsic reaction coordinate (IRC) towards the transition state for the second mechanistic step. Although this transition state emphasizes motion of the second proton, some displacement in position of both protons is seen. If the $\mathrm{N}$ to $\mathrm{O}$ distance is large when transfer occurs, then 106-B takes place with two distinct transition states, though the potential landscape is still relatively flat with a shallow minimum defining the intermediate. At a collision energy of $8 \mathrm{eV}$, the fraction of $\mathrm{m} / \mathrm{z} 106$ that results from Mechanism 106-B is 0.079. DFT calculations show that this mechanism has a barrier of $44.1 \mathrm{kcal} / \mathrm{mol}$ and involves the migration of two protons.

The carbonyl oxygen of the C-terminus can also be involved in the generation of the protonated serine ion, though it is much less common. Mechanism 106-C involves proton transfers from the $\mathrm{N}$ - terminus to the oxygen of the side chain and from the sulfate group to the carbonyl oxygen. This step is followed by a transfer from the C-terminus to the $\mathrm{N}$-terminus to generate $\mathrm{N}$-protonated serine and $\mathrm{SO}_{3}$. DFT calculations show that these three proton transfer reactions occur along an IRC that involves motion of all three protons and has a barrier of $29.9 \mathrm{kcal} / \mathrm{mol}$. Mechanism $106-\mathrm{C}$ is not observed for most collision energies, but does account for 0.014 of $\mathrm{m} / \mathrm{z} 106$ at a collision energy of $6.5 \mathrm{eV}$. Its lack of prevalence in the simulations is likely due to the large number of protons that need to be exchanged, and hence depends sensitively on the overall conformation of the system.

The second class of mechanism involves intermolecular proton transfer reactions. These mechanisms typically begin along the same reaction pathway as 106-B and C, namely a proton transfer from the N-terminus to the linking oxygen. This transfer results in the formation of $\mathrm{HSO}_{3}^{+}$ along with neutral serine. A complex then forms between the charged and neutral fragmentation products. The formation of such complexes between fragmentation products has previously been studied by Barnes and co-workers ${ }^{24}$ for the octaglycine system. In that system, it was found that complexes could be very long lived and allow for unique final products. In this system, the complexes can be long lived or fleeting, depending on the proton motion taking place in the rest of the system along with the conformation of the system when the S-O bond cleaves. The final $\mathrm{m} / z, 106$ product is generated by the transfer of the proton from $\mathrm{HSO}_{3}^{+}$to serine. Multiple different acceptor sites have been observed, though it is common for the proton to transfer to the side-chain alcohol, which simultaneously transfers the proton that was already at that location back to the $\mathrm{N}$-terminus. A similar type of mechanism has been observed at the C-terminus; transfer occurs to the carbonyl oxygen along with a simultaneous transfer of the acidic proton back to the $\mathrm{N}$-terminus. This class of mechanism is significant and has a maximum contribution to $\mathrm{m} / \mathrm{z}, 106$ of 0.265 at a collision energy of $5 \mathrm{eV}$.

The final class for $m / z, 106$ is shattering mechanisms, which are both rare and only occur at large collision energies. As such only qualitative comments can be made. This class of reaction mecha- 


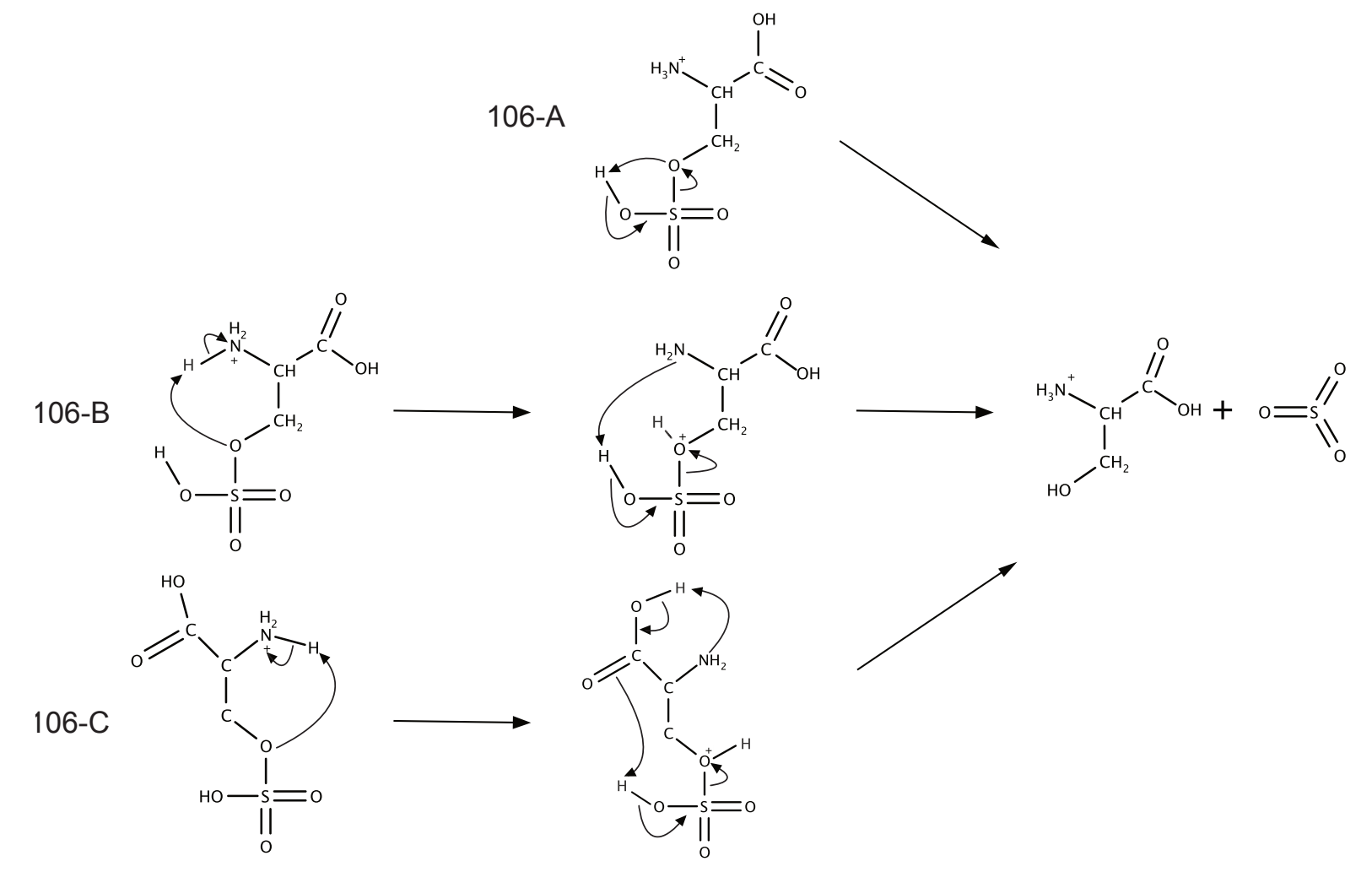

Scheme 1: The mechanisms for $m / z, 106$.

nism produces $m / z$ 106, but not protonated serine. Rather, these trajectories result in the loss of multiple small molecules and one charged cyclic product, namely $\mathrm{H}_{2} \mathrm{O}+\mathrm{CO}+\mathrm{c}\left((\mathrm{S}=\mathrm{O}) \mathrm{OCH}_{2} \mathrm{CHN}^{+} \mathrm{H}\right)$ with the $\mathrm{S}$ atom within the 5 -member ring structure.

\subsubsection{Sulfoserine $m / z, 88$}

We performed the same connectivity analysis to determine the structure of the $m / z 88$ fragment ion. However, unlike the majority of the products for the $m / z 106$ ion, the $m / z 88$ peak was observed to consist of several different constitutional isomers. In fact, 11 different products were identified for this peak. These products were efficiently sorted and quantified by using the connectivity information collected during the trajectory combined with group theoretical analysis - it is known that the lowest eigenvalue of the weighted adjacency matrix uniquely determines the particular constitutional isomer. ${ }^{34,35}$ Of the possible product ions, all but three are minor pathways within our simulation. The mechanisms for the formation of these products is given in Scheme 2 and their relative contribution to the $m / z 88$ peak is shown in Figure 3. Many of the other eight structures are seen at large collision energies and result from backbone rearrangement reactions.

Product 1 is seen to be most important in the simulations for all collision energies, starting at a fraction of 1 for $2 \mathrm{eV}$ and moving down to $\approx 0.75$ at $11 \mathrm{eV}$ with Products 2 and 3 increasing to 0.17 and 0.07 , respectively. Polfer and coworkers proposed five different possible structures for $m / z 88$ that occur either via direct loss of $\mathrm{H}_{2} \mathrm{SO}_{4}$ or loss of $\mathrm{SO}_{3}$ followed by loss of $\mathrm{H}_{2} \mathrm{O}$. The proposed products included our Product 1 and 2, but not 3; we observed three of the five proposed structures. In contrast to Polfer, who assigned Product 2 to be the most likely structure, we observed Product 1 as most important in our short time simulations. This may be due to the short timescale of the simulations, or that PM6 underestimates the reaction barrier for this process.

Our DFT calculations provide information regarding the reaction pathways that produce these 

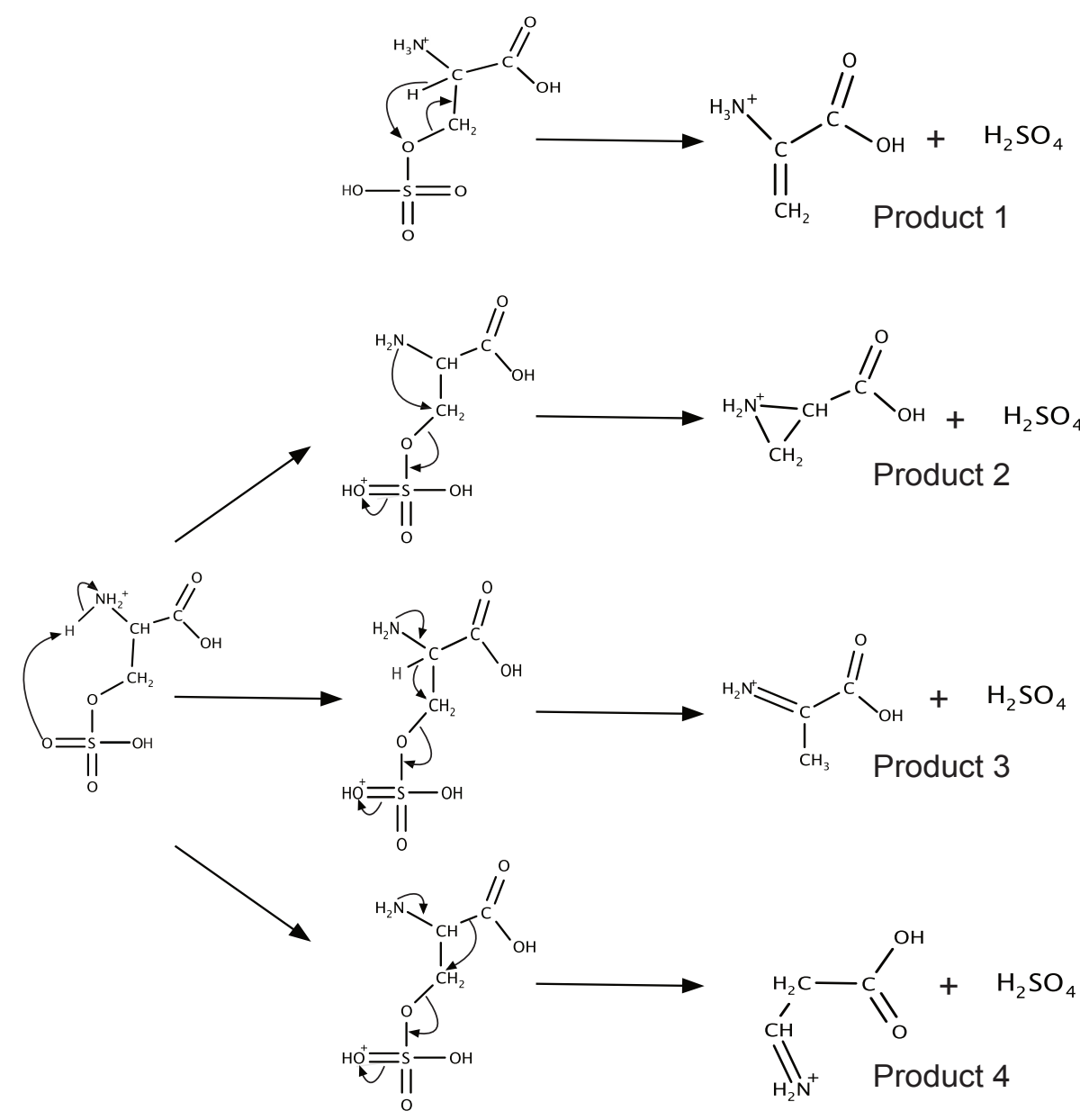

Scheme 2: The mechanisms for $m / z 88$.

products as well as insight into the trends observed in Figure 3. A schematic overview of the reaction pathways is provided in Figure 4. The mechanism for the formation of Product 1 follows a direct, one-step process to release $\mathrm{H}_{2} \mathrm{SO}_{4}$ via loss of the alpha carbon's hydrogen atom. This mechanism is slightly different than that proposed by Polfer in that the linking oxygen to the sulfo group is not involved. Product 2 is generated from a two-step mechanism involving an intermediate that results from a proton transfer from the $\mathrm{N}$-terminus to the sulfate group. In the global minima, the proton that is transferred is hydrogen bonded to the sulfate group, but in the intermediate, following the transfer, this proton is stabilized by a hydrogen bond to the C-terminus carbonyl group. There is no barrier for a return to the global minima, and hence the system readily reverts. This reversibility and the underestimate of Product 1's barrier likely accounts for the populations observed in the sim- ulations. Product 3, which also involves a proton transfer from the N-terminus to the sulfo group, forms directly from the global minima, does not pass through the stabilized intermediate observed for Product 2, and increases with collision energy monotonically. This is consistent with Product 3's higher barrier but more stable final structure. The reaction barriers at the PM6 level of theory for both Products 2 and 3 are within $3 \mathrm{kcal} / \mathrm{mol}$ of the DFT values. The DFT calculations for Products 1-3 also yielded the pathway for Product 4. While Product 4 is observed in the simulations, it is not a major product. However, based on the DFT calculations, it could be important. The PM6 barrier for this TS is $65.2 \mathrm{kcal} / \mathrm{mol}$, which is $15.7 \mathrm{kcal} / \mathrm{mol}$ higher than in the DFT calculations, which could explain why it is not seen in the PM6 simulations. While the mechanism involves a proton transfer from the N-terminus to the sulfate group, it proceeds directly to products. 


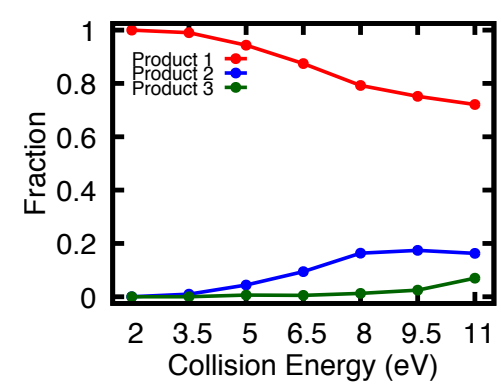

Figure 3: The time evolution for the distribution of products observed within the $m / z 88$ peak. While Product 1 is dominant throughout the entire collision energy range, it is seen to decrease with collision energy as competition from other products increases.

The experimental assignment of the $m / z, 88$ ion as Product 2 two was based on IRMPD measurements compared to DFT frequency calculations. ${ }^{17}$ In addition, the product resulting from loss of phosphoric acid from phosphoserine has also been identified as 2-carboxy-aziridine via both traditional MS methods ${ }^{12}$ and IRMPD measurements. ${ }^{13}$ However, the DFT calculations here suggest that our Product 4 could also be important. Hence, we decided to closely compare the theoretical IR spectra for each ion. Katari et al ${ }^{40}$ have recently shown that use of a linear correlation rather than a simple scaling improves mean absolute error for experimental vs. scaled frequencies, and in particular focused on comparison to IRMPD data. Hence, we re-optimized both Products 2 and 4 using the B3LYP/6-31+G(d,p) level of theory and obtained their vibrational frequencies. Katari found that this choice of functional and basis set produced the best results. Lorentzian line shapes with $30 \mathrm{~cm}^{-1}$ FWHM were applied to each and presented in Figure 5 along with dashed lines showing the centers of the most important experimental peaks as identified by Polfer and coworkers. Product 2 is likely the better match to the observed IRMPD spectrum; however, the two are fairly close and it is notable that Product 4 does not have a peak at $\approx 3200 \mathrm{~cm}^{-1}$, but rather one at $\approx 2988 \mathrm{~cm}^{-1}$, which is out of the experimentally observable range. Product 2 has the lowest energy reaction pathway whereas Product 4 is the most thermodynamically stable. Thus, it is plau-

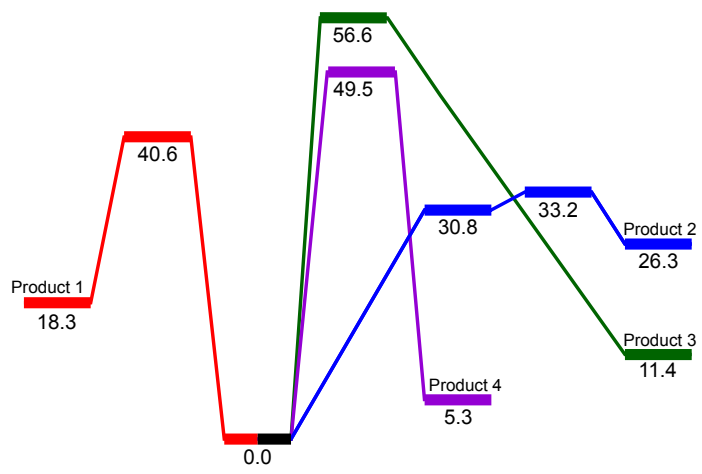

Figure 4: A schematic overview of the energy landscape for Products 1-4 along with the energy, in $\mathrm{kcal} / \mathrm{mol}$, of the relevant minima, intermediates, products, and transition states at the $\omega \mathrm{B} 97 \mathrm{X}$ D/aug-cc-pVTZ level of theory.

sible that either could dominate depending on the experimental conditions.

\subsubsection{Sulfoserine $m / z 81$}

The $m / z 81$ peak is seen to dramatically rise in abundance, specifically as the other three ion intensities slowly taper. While this peak was not observed by Polfer and co-workers, it is possible that it was not present at the collision energy considered in that experiment. The $m / z 81$ peak corresponds to the formation of $\mathrm{HSO}_{3}^{+}$. One obvious source for this peak has already been discussed; a lack of proton transfer from $\mathrm{HSO}_{3}^{+}$back to serine which results in $m / z, 81$ rather than $m / z$ 106. At a collision energy of $11 \mathrm{eV}$, this pathway accounts for a 0.53 fraction of the $\mathrm{m} / \mathrm{z} 81$ peak. Another major pathway, with a 0.35 fraction of the peak, is the complete loss of the side-chain. The side-chain itself then fragments to form $\mathrm{HSO}_{3}^{+}+\mathrm{CH}_{2} \mathrm{O}$, without any proton transfer occurring between the initial fragmentation products. The fragments of the side-chain often complex; however, for this peak, that complex is broken without charge transfer occurring. The next most favored pathway, with a fraction of 0.05 , follows the same steps with the addition of water loss occurring at what was the Cterminus. The remaining components of the peak are distributed among eight other minor product pathways. 


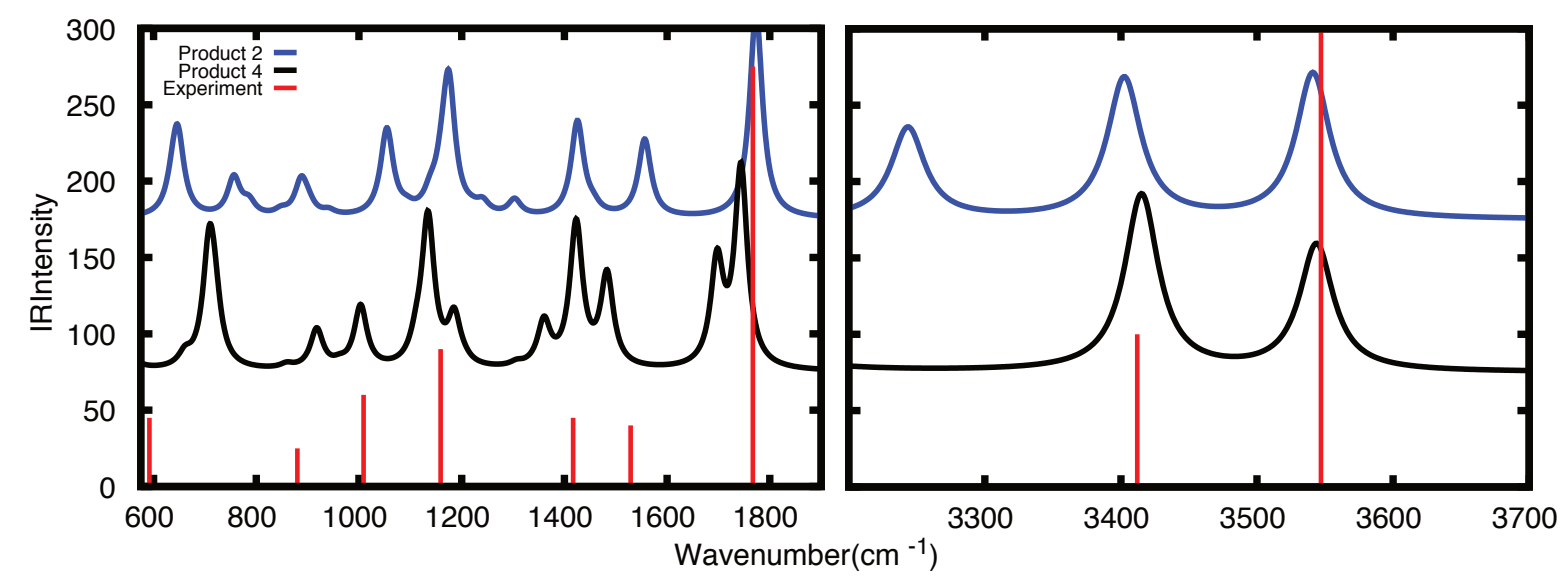

Figure 5: A comparison of the DFT frequencies for Products 2 and 4 along with the major experimental peaks identified by Polfer and co-workers. Experimental intensities are estimated, while theoretical peaks are obtained from a linear correlation correction and a $30 \mathrm{~cm}^{-1} \mathrm{FWHM}$ Lorentzian line shape.

\section{Summary}

We have presented results from direct dynamics simulations and DFT calculations that highlighted the differences between unmodified serine and sulfoserine. In general, the direct dynamics simulations show good qualitative agreement with the experimental work of Zhang et al (serine) ${ }^{37}$ as well as Polfer and co-workers (sulfoserine). ${ }^{17}$ Transition state searches, geometry optimizations, and IRC calculations were performed to elucidate the potential energy surface for sulfoserine based on the products observed from the dynamics simulations.

The major peaks observed in the sulfoserine experiment were $\mathrm{m} / \mathrm{z} 106$ and $\mathrm{m} / \mathrm{z} 88$. Detailed analysis was performed on these peaks. The $\mathrm{m} / \mathrm{z} 106$ peak was found to be formed through three different classes of mechanism: intramolecular rearrangement, intermolecular proton transfer reactions, and "shattering" processes. The dominant pathway involves a direct, one-step mechanism that was also put forward by Polfer and coworkers. Our work highlights that other rearrangement mechanisms are possible, but in addition, intermolecular mechanisms involving proton transfer from initial fragmentation products were observed as a significant pathway for this peak.

The composition of the $m / z 88$ peak was ana- lyzed and compared to the findings of Polfer and co-workers. The dynamics simulations show that Product 1 is the favored isomer for all collision energies and times considered; however, this is likely due to the underestimation of the reaction barrier by PM6. Product 2, which was identified by Polfer as the dominant species, was seen to increase with collision energy. DFT calculations also show that based on energetics, Product 4 may also be important, although it is a minor pathway in simulations due to the overestimation of this reaction barrier by PM6 compared to the DFT results. The other barriers for $m / z 88$ were well reproduced by PM6. The pathway that forms Product 2 has the lowest reaction barriers, while Product 4 is the most thermodynamically stable. A detailed comparison of the vibrational frequencies is provided for both of these products. While Product 2 is likely a better match for the experimental IRMPD measurements of Polfer, the two species share many spectral features.

The $\mathrm{m} / \mathrm{z} 81$ feature is observed in simulations, but not in the experimental work. This may be due to the energy at which the experiment was conducted. The $\mathrm{m} / \mathrm{z} 81$ and $\mathrm{m} / \mathrm{z} 106$ peaks are in fact linked in that the intermolecular proton transfer mechanisms that produce $\mathrm{m} / \mathrm{z}, 106$ could also result in $\mathrm{m} / \mathrm{z} 81$ if the complex breaks before proton transfer occurs. This is consistent with $m / z 81$ 
being more prevalent at high collision energies. In addition, $m / z 81$ arises through side-chain loss followed by fragmentation of the side-chain itself, which is again a high energy process.

\section{ACKNOWLEDGEMENTS}

GLB and KL gratefully acknowledge support from the National Science Foundation under grant No. 1763652. Dr. K. Kolonko is thanked for insightful conversations.

\section{References}

(1) Cooks, R. G.; Ast, T.; Pradeep, T.; Wysocki, V. H. Reactions of Ions with Organic Surfaces. Acc. Chem. Res. 1994, 27, 316-323.

(2) Ouyang, Z.; Takáts, Z.; Blake, T. A.; Gologan, B.; Guymon, A. J.; Wiseman, J. M.; Oliver, J. C.; Davisson, V. J.; Cooks, R. G. Preparing Protein Microarrays by SoftLanding of Mass-Selected Ions. Science 2003, 301, 1351-1354.

(3) Laskin, J.; Denisov, E.; Futrell, J. H. A Comparative Study of Collision-Induced and Surface-Induced Dissociation. 1. Fragmentation of Protonated Dialanine. J. Am. Chem. Soc. 2000, 122, 9703-9714.

(4) Laskin, J.; Wang, P.; Hadjar, O. Soft-Landing of Peptide Ions Onto Self-Assembled Monolayer Surfaces: An Overview. Phys. Chem. Chem. Phys. 2008, 10, 1079-1090.

(5) Wang, P.; Hadjar, O.; Gassman, P. L.; Laskin, J. Reactive Landing of Peptide Ions on Self-Assembled Monolayer Surfaces: An Alternative Approach for Covalent Immobilization of Peptides on Surfaces. Phys. Chem. Chem. Phys. 2008, 10, 1512-1522.

(6) Pratihar, S.; Barnes, G. L.; Hase, W. L.; Pratihara, S.; Barnes, G. L.; Hase, W. L. Chemical Dynamics Simulations of Energy Transfer, Surface-Induced Dissociation, Soft-Landing, and Reactive- Landing in Collisions of Protonated Peptide Ions with Organic Surfaces. Chem. Soc. Rev. 2015, 45, 3595-3608.

(7) Pratihar, S.; Barnes, G. L.; Laskin, J.; Hase, W. L. Dynamics of Protonated Peptide Ion Collisions with Organic Surfaces. Consonance of Simulation and Experiment. J. Phys. Chem. Lett. 2016, 7, 3142-3150.

(8) Kumar, A.; Narayanan, V.; Sekhar, A. Characterizing Post-Translational Modifications and Their Effects on Protein Conformation Using NMR Spectroscopy. Biochemistry 2019, 59, 57-73.

(9) Ryšlavá, H.; Doubnerová, V.; Kavan, D.; Vaněk, O. Effect of posttranslational modifications on enzyme function and assembly. J. Proteomics 2013, 92, 80-109.

(10) Boscher, C.; Dennis, J. W.; Nabi, I. R. Glycosylation, galectins and cellular signaling. Curr. Opin. Cell Biol. 2011, 23, 383-392.

(11) Karve, T. M.; Cheema, A. K. Small Changes Huge Impact: The Role of Protein Posttranslational Modifications in Cellular Homeostasis and Disease. J. Amino Acids 2011, 2011, 207691(1-13).

(12) Reid, G. E.; Simpson, R. J.; O’Hair, R. A. Leaving group and gas phase neighboring group effects in the side chain losses from protonated serine and its derivatives. J. Am. Soc. Mass Spectrom. 2000, 11, 1047-1060.

(13) Lanucara, F.; Chiavarino, B.; Scuderi, D.; Maitre, P.; Fornarini, S.; Crestoni, M. E. Kinetic control in the CID-induced elimination of $\mathrm{H}_{3} \mathrm{PO}_{4}$ from phosphorylated serine probed using IRMPD spectroscopy. Chem. Commun. 2014, 50, 3845-3848.

(14) Medzihradszky, K. F.; Darula, Z.; Perlson, E.; Fainzilber, M.; Chalkley, R. J.; Ball, H.; Greenbaum, D.; Bogyo, M.; Tyson, D. R.; Bradshaw, R. A.; Burlingame, A. L. O-sulfonation of serine and threonine. Mol. Cell. Proteomics 2004, 3, 429-443. 
(15) Seibert, C.; Sakmar, T. P. Toward a framework for sulfoproteomics: Synthesis and characterization of sulfotyrosine-containing peptides. 2008; http://doi.wiley.com/ 10.1002 /bip. 20821.

(16) Huang, B. Y.; Chen, P. C.; Chen, B. H.; Wang, C. C.; Liu, H. F.; Chen, Y. Z.; Chen, C. S.; Yang, Y. S. High-Throughput Screening of Sulfated Proteins by Using a Genome-Wide Proteome Microarray and Protein Tyrosine Sulfation System. Anal. Chem. 2017, 89, 3278-3284.

(17) Patrick, A. L.; Stedwell, C. N.; Schindler, B.; Compagnon, I.; Berden, G.; Oomens, J.; Polfer, N. C. Insights into the fragmentation pathways of gas-phase protonated sulfoserine. Int. J. Mass Spectrom. 2014,

(18) Martin Somer, A.; Macaluso, V.; Barnes, G. L.; Yang, L.; Pratihar, S.; Song, K.; Hase, W. L.; Spezia, R. Role of Chemical Dynamics Simulations in Mass Spectrometry Studies of Collision-Induced Dissociation and Collisions of Biological Ions with Organic Surfaces. J. Am. Soc. Mass Spectrom. 2020, 31, 2-24.

(19) Barnes, G. L.; Hase, W. L. Energy Transfer, Unfolding, and Fragmentation Dynamics in Collisions of N-Protonated Cctaglycine with an H-SAM Surface. J. Am. Chem. Soc. 2009, 131, 17185-17193.

(20) Frederickson, D.; McDonough, M.; Barnes, G. L. A Computational Comparison of Soft Landing of Alpha-Helical vs Globular Peptides. J. Phys. Chem. B 2018, 122, 9549-9554.

(21) Barnes, G. L.; Podczerwinski, A. Simulating the Effect of Charge State on Reactive Landing of a Cyclic Tetrapeptide on Chemically Modified Alkylthiolate Self-Assembled Monolayer Surfaces. J. Phys. Chem. C 2017, 121, 14628-14635.

(22) Shaikh, K.; Blackwood, J.; Barnes, G. L. The Effect of Protonation Site and Conformation on Surface-Induced Dissociation in a Small, Lysine Containing Peptide. Chem. Phys. Lett. 2015, 637, 83-87.

(23) Gregg, Z.; Ijaz, W.; Jannetti, S.; Barnes, G. L. The Role of Proton Transfer in SurfaceInduced Dissociation. J. Phys. Chem. C 2014, 118, 22149-22155.

(24) Ijaz, W.; Gregg, Z.; Barnes, G. L. Complex Formation during SID and Its Effect on Proton Mobility. J. Phys. Chem. Lett. 2013, 4, 3935-3939.

(25) Geragotelis, A.; Barnes, G. L. Surface Deposition Resulting from Collisions Between Diglycine and Chemically Modified Alkylthiolate Self-Assembled Monolayer Surfaces. J. Phys. Chem. C 2013, 117, 13087-13093.

(26) Macaluso, V.; Scuderi, D.; Crestoni, M. E.; Fornarini, S.; Corinti, D.; Dalloz, E.; Martinez-Nunez, E.; Hase, W. L.; Spezia, R. L -Cysteine Modified by S-Sulfation: Consequence on Fragmentation Processes Elucidated by Tandem Mass Spectrometry and Chemical Dynamics Simulations. J. Phys. Chem. A 2019, 123, 3685-3696.

(27) Meroueh, O.; Hase, W. L. Collisional Activation of Small Peptides. J. Phys. Chem. A 1999, 103, 3981-3990.

(28) Ortiz, D.; Salpin, J.-Y.; Song, K.; Spezia, R. Galactose-6-Sulfate collision induced dissociation using QM+MM chemical dynamics simulations and ESI-MS/MS experiments. Int. J. Mass Spectrom. 2014, 358, 25-35.

(29) Hanwell, M. D.; Curtis, D. E.; Lonie, D. C.; Vandermeersch, T.; Zurek, E.; Hutchison, G. R. Avogadro: An Advanced Semantic Chemical Editor, Visualization, and Analysis Platform. J. Cheminform. 2012, $\underline{4}, 17$.

(30) Stewart, J. P. Mopac2016. 2016; http:// openmopac.net.

(31) Peslherbe, G. H.; Wang, H.; Hase, W. L. Monte Carlo Sampling for Classical Trajectory Simulations. Adv. Chem. Phys. 1999, 105, 171-201. 
(32) Schlier, C.; Seiter, A. High-Order Symplectic Integration: An Assessment. Comput. Phys. Commun. 2000, 130, 176-189.

(33) Stewart, J. P. Mopac2012. 2012; http:// openmopac. net.

(34) Vázquez, S. A.; Otero, X. L.; MartinezNunez, E. A trajectory-based method to explore reaction mechanisms. 2018; http:// www. mdpi.com/1420-3049/23/12/3156.

(35) Rodríguez, A.; Rodríguez-Fernández, R.; A. Vázquez, S.; L. Barnes, G.; J. P. Stewart, J.; Martínez-Núñez, E. tsscds2018: A code for automated discovery of chemical reaction mechanisms and solving the kinetics. J. Comput. Chem. 2018, 39, 1922-1930.

(36) Frisch, M. J. et al. Gaussian 09, Revision D.01. 2009.

(37) Zhang, P.; Chan, W.; Ang, I. L.; Wei, R.; Lam, M. M.; Lei, K. M.; Poon, T. C. Revisiting Fragmentation Reactions of Protonated $\alpha$-Amino Acids by High-Resolution Electrospray Ionization Tandem Mass Spectrometry with Collision-Induced Dissociation. Sci. Rep. 2019, 9, 6453.

(38) Humbel, S. Short strong hydrogen bonds: A valence bond analysis. J. Phys. Chem. A 2002, 106, 5517-5520.

(39) Vishveshwara, S.; Madhusudhan, M. S.; Maizel, J. V. Short-strong hydrogen bonds and a low barrier transition state for the proton transfer reaction in RNase A catalysis: A quantum chemical study. Biophys. Chem. 2001, 89, 105-117.

(40) Katari, M.; Nicol, E.; Steinmetz, V.; van der Rest, G.; Carmichael, D.; Frison, G. Improved Infrared Spectra Prediction by DFT from a New Experimental Database. Chem. A Eur. J. 2017, 23, 8414-8423. 